\title{
Energy dissipation and flux laws for unsteady turbulence
}

\author{
Susumu Goto \\ Graduate School of Engineering Science, Osaka University, 1-3 Machikaneyama, \\ Toyonaka, Osaka, 560-8531 Japan \\ J. C. Vassilicos \\ Department of Aeronautics, Imperial College, London, SW7 2AZ, UK
}

\begin{abstract}
Direct Numerical Simulations of unsteady spatially periodic turbulence with time-dependent rms velocity $u^{\prime}(t)$ and integral length-scale $L(t)$ show that not only the instantaneous energy dissipation rate but also the instantaneous energy flux at intermediate wavenumbers scales as $U_{0} L_{0} u^{\prime}(t)^{2} / L(t)^{2}$ where $U_{0}$ and $L_{0}$ are velocity and length scales characterizing initial or overall unsteady turbulence conditions. These high Reynolds number scalings are qualitatively different from the well-known $u^{\prime}(t)^{3} / L(t)$ cornerstone scalings of equilibrium turbulence where the energy flux and dissipation are exactly balanced at all times.
\end{abstract}

Keywords: Turbulence, Energy Dissipation, Energy Flux, Direct

Numerical Simulation

PACS: 47.27.Ak, 47.27.Jv, 47.27.Gs, 47.27.eb

\section{Introduction}

Recent laboratory experiments (see Ref. [1] and references therein) show that in a variety of decaying turbulent flows with different levels of statistical homogeneity and a wavenumber dependence of the energy spectrum $E(k, t)$ close to $k^{-5 / 3}$, the following law of the dissipation rate $\epsilon(t)$ of turbulent kinetic

\footnotetext{
Email addresses: goto@me.es.osaka-u.ac.jp (Susumu Goto), j.c.vassilicos@imperial.ac.uk (J. C. Vassilicos)
} 
energy is observed:

$$
\epsilon(t) \sim \frac{R e_{0}^{p / 2}}{R e_{\lambda}(t)^{q}} \frac{u^{\prime}(t)^{3}}{L(t)}
$$

where $p \approx 1, q \approx 1, t$ is time, $L(t)$ is an integral length-scale, $R e_{0}=U_{0} L_{0} / \nu$ is a global Reynolds number based on a velocity $U_{0}$ and a length scale $L_{0}$ characterizing the initial/inlet conditions, $R e_{\lambda}=u^{\prime} \lambda / \nu$ is a local Reynolds number based on the Taylor length $\lambda(t)$ and the rms turbulence velocity $u^{\prime}(t)$, and $\nu$ is the kinematic viscosity of the fluid. In wind tunnel experiments, the variables $u^{\prime}(t), L(t)$ and $\epsilon(t)$ are effectively ensemble averaged over many realizations and the time $t$ is defined so that $U_{0} t$ corresponds to the distance from the grid. In our numerical simulations, however, these variables are spatially averaged and are functions of time $t$ as they vary in time. This dissipation law (1) is fundamentally different from the well-known scaling first introduced by Taylor [2]

$$
\epsilon(t)=C_{\epsilon} \frac{u^{\prime}(t)^{3}}{L(t)}
$$

where $C_{\epsilon}$ is a dimensionless time-independent constant. This law (2) of dissipation relates $\epsilon(t)$, which is a small-scale quantity, to the large-scale flow properties $L(t)$ and $u^{\prime}(t)$ and has therefore provided a foundation for the modeling of small-scale turbulence in very many contexts (see, for example, Refs. $[3,4,5])$.

As explained in Ref. [1], whereas (2) is compatible with equilibrium turbulence where the energy flux and dissipation are exactly balanced at all times, the dissipation scaling (1) is not. This paper's objectives are, firstly, to show that the new dissipation law (1) also holds in Direct Numerical Simulations (DNS) of two very different kinds of unsteady spatially periodic turbulence at high enough Reynolds number and, secondly and much more importantly, to demonstrate that in these two unsteady turbulent flows, the interscale energy flux at intermediate wavenumbers scales in the same way as the dissipation at high enough Reynolds number.

To fully appreciate the relevance of these two objectives it may be worth recalling that the scale-by-scale energy balance in spatially periodic turbulence is the same as in homogeneous (not necessarily isotropic) turbulence (see Ref. [6]). In spectral space, this balance is the Lin equation (see Ref. $[7,6,8])$

$$
\frac{\partial}{\partial t} E(k, t)=-\frac{\partial}{\partial k} \Pi(k, t)-2 \nu k^{2} E(k, t)
$$


where $\Pi(k, t)$ is the interscale energy flux to Fourier modes with wavenumber larger than wavenumber $k$. Kolmogorov's pivotal local stationarity assumption [9] implies $\partial E / \partial t=0$ for $k \gg 1 / L$. Integrating (3) in such a wavenumber range, from a given wavenumber $k(\ll 1 / \eta$ where $\eta$ is the Kolmogorov length-scale) to $\infty$ and using $\epsilon(t)=2 \nu \int_{0}^{\infty} k^{2} E(k, t) \mathrm{d} k$ and $\Pi(\infty, t)=0$, one obtains $\Pi(k, t) \approx \epsilon(t)$. Note that the wavenumber range $1 / L \ll k \ll 1 / \eta$ exists only when the Reynolds number is sufficiently high. The local stationarity and the resulting local balance between energy flux and dissipation are a cornerstone of the equilibrium energy cascade in the so-called inertial range $(1 / L \ll k \ll 1 / \eta)$, and the reason why $\epsilon(t)$ is the key quantity in Kolmogorov's similarity hypotheses [9]. Returning to our objectives, our first one is to check that (1) also holds in an unsteady turbulence where the interscale balance equation (3) is demonstrably the same as in homogeneous turbulence. And our most important second objective is to examine $\Pi(k, t)$ in such unsteady turbulence and show how it scales at intermediate wavenumbers. Kolmogorov's stationarity assumption applied to the Kármán-Howarth equation (the equivalent of the Lin equation in physical space) leads to (2) (see Ref. [1] where the arguments leading to this conclusion are given without need for statistical isotropy). Given that $\Pi(k, t) \approx \epsilon(t)$ under the local stationarity assumption in intermediate wavenumbers, it then follows that $\Pi(k, t)$ also scales as $u^{\prime}(t)^{3} / L(t)$. However, what happens to $\Pi(k, t)$ if $\epsilon(t)$ does not scale as $u^{\prime}(t)^{3} / L(t)$ but instead obeys (1) as recently observed in many laboratory experiments [1] of various decaying turbulent flows? Although arguably the scaling of $\Pi(k, t)$ is even more important than that of $\epsilon(t)$ because $\Pi(k, t)$ is central to coarse-graining approaches to turbulent flows such as Large Eddy Simulations, only a few authors $[10,11]$ investigated the scaling of $\Pi(k, t)$. This is the motivation for our second, and in fact major, objective.

\section{Energy dissipation law}

Let us start with demonstrating that (1) is also observed in DNS of unsteady turbulence in a periodic cube. Before doing this, we derive equivalent forms of (1). Firstly, combining with the expression [2] $\epsilon(t)=15 \nu u^{\prime}(t)^{2} / \lambda(t)^{2}$ for isotropic turbulence, and assuming $p=q=1$ for simplicity, (1) becomes

$$
\epsilon(t)=D_{\epsilon} U_{0} L_{0} \frac{u^{\prime}(t)^{2}}{L(t)^{2}}
$$


where $D_{\epsilon}$ is a dimensionless constant. Next, regarding (2) as the definition of $C_{\epsilon}$, it is seen that (1) is equivalent to

$$
C_{\epsilon}(t) \sim \frac{\sqrt{R e_{0}}}{R e_{\lambda}(t)}
$$

for $p=q=1$. Now we verify (5) numerically.

We conducted DNS of turbulent incompressible fluid flows in a periodic cube. We used a Fourier spectral code with de-aliasing by the phase shift method and conducted both decaying and forced simulations. The forcing ${ }^{1}$ imposed on the Navier-Stokes equation was

$$
\boldsymbol{f}=\left[\begin{array}{c}
+\sin (2 \pi m x / \mathcal{L}) \cos (2 \pi m y / \mathcal{L}) \\
-\cos (2 \pi m x / \mathcal{L}) \sin (2 \pi m y / \mathcal{L}) \\
0
\end{array}\right]
$$

where $\mathcal{L}$ is the spatial period of the boundary condition and $m$ is an integer. For the decaying turbulence cases we chose $m=4$ so that $L(t)$ is sufficiently smaller than $\mathcal{L}$ (i.e. $L(t)<0.1 \mathcal{L})$ during the decay, and switched off the force when the dissipation rate $\epsilon(t)$ reached its maximum value. We considered five different values of $R e_{0}$ corresponding to simulation sizes between $128^{3}$ and $2048^{3}$ so that the smallest eddies are always well resolved. More concretely, the values of the kinematic viscosity were chosen so that $k_{\max } \eta$ (where $k_{\max }$ is the largest resolved wavenumber) is larger than 1 throughout the decay. The results are shown in Fig. 1; as time advances and the turbulence decays, $R e_{\lambda}$ decays but $C_{\epsilon}$ grows and $C_{\epsilon}(t)$ is approximately proportional to $R e_{\lambda}(t)^{-1}$. The different $C_{\epsilon}(t) \propto R e_{\lambda}(t)^{-1}$ curves collapse very well if we plot $C_{\epsilon}(t) / \sqrt{R e_{0}}$ as a function of $\operatorname{Re}_{\lambda}(t)$ (insert of Fig. 1) in good agreement with (5), i.e. the dissipation law (1) with $p=q=1$ and $U_{0}, L_{0}$ given by the rms turbulent velocity and the integral scale at the initial time of decay. The normalized time duration of each run along each one of these curves is estimated by the temporal integration of $1 / T(t)$ (where $T(t)=L(t) / u^{\prime}(t)$ is the

\footnotetext{
${ }^{1}$ This force drives anisotropic turbulence at large scales (as is often the case, in fact, both in nature and industry). The temporal averages of the rms values of velocity components are $\left\langle u_{1}^{\prime}\right\rangle \approx\left\langle u_{2}^{\prime}\right\rangle \approx 1.6\left\langle u_{3}^{\prime}\right\rangle$ when the force is continuously applied. The turbulence is, however, statistically isotropic at small scales even for lower Reynolds number cases in the sense that the rms values of the three components of vorticity are identical within statistical error.
} 


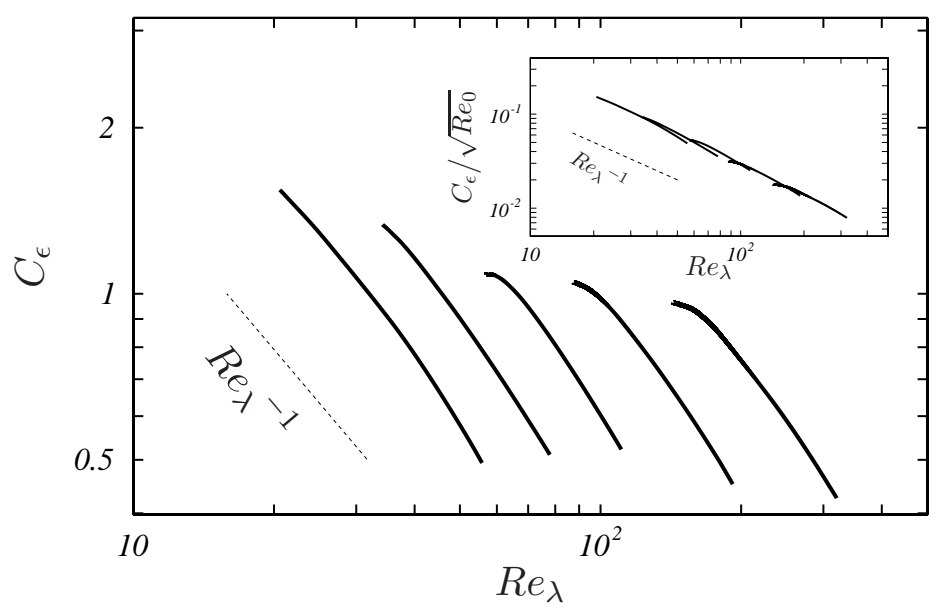

Figure 1: Instantaneous value of $C_{\epsilon}(t)$ as a function of $R e_{\lambda}(t)$ in the decaying turbulence. Five curves are plotted for five different values of $\nu$. Insert: $C_{\epsilon}(t)$ normalized by $\sqrt{R e_{0}}$.

eddy turnover time) and is 3.2, 3.2, 3.2, 4.2 and 5.0 in order of increasing $R e_{0}$. For longer times (not shown in Fig. 1), $C_{\epsilon}$ tends towards a constant for a little while as $R e_{\lambda}$ decays further till $R e_{\lambda}$ eventually reaches values low enough for the dissipation to be directly viscous. The behavior of $C_{\epsilon}$ in later stages of turbulence decay is beyond the scope of this study. However, it is worth mentioning that Meldi et al.[12] studied the decay of very high Reynolds number turbulence by using an EDQNM model and showed that $C_{\epsilon}$ tends to a constant in a later stage of decay. Their observation is consistent with wind tunnel observations in the far downstream region of grid-generated turbulence (see Ref. [1] and references therein).

The range of $R e_{\lambda}$ values in our decaying simulations (Fig. 1) is rather limited and under $R e_{\lambda}=400$ in all cases. To conduct DNS at higher Reynolds numbers, we used (6) with $m=1$ and kept the forcing on throughout. We considered seven different values of $R e_{0}$ corresponding to simulation sizes between $64^{3}$ and $2048^{3}$ for similar resolutions: $k_{\max }\langle\eta\rangle=1.7,1.0,1.1,1.4$, $1.4,1.4$ and 1.3, respectively. Here, brackets $\langle\cdot\rangle$ denote the temporal average. Interestingly, the turbulence driven by this steady force is far from steady and $u^{\prime}(t), L(t)$ and $\epsilon(t)$ oscillate significantly in time with a time scale of about $20\langle T\rangle$, where $\langle T\rangle=\left\langle L(t) / u^{\prime}(t)\right\rangle$. This quasi-periodic behavior of the forced turbulence is evident in Fig. 2 where the temporal evolution of $u^{\prime}(t), L(t), \epsilon(t), \operatorname{Re}_{\lambda}(t)$ and $C_{\epsilon}(t)$ is plotted for a given $R e_{0}$. Note that the global Reynolds number $R e_{0}$ is redefined for the forced turbulence in terms 


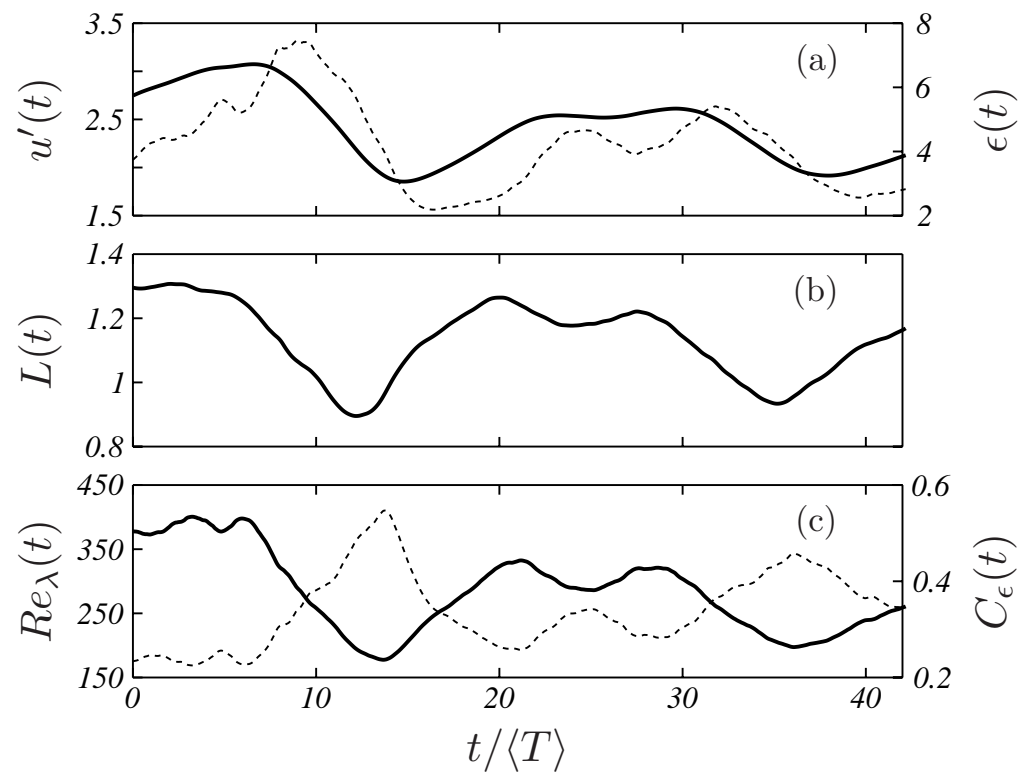

Figure 2: Temporal evolution of (a) $u^{\prime}(t)$ (solid line), $\epsilon(t)$ (dotted line), (b) $L(t),\left(\right.$ c) $R e_{\lambda}(t)$ (solid line) and $C_{\epsilon}(t)$ (dotted line) in the forced turbulence at the average Reynolds number $\left\langle R e_{\lambda}\right\rangle=280$. The origin of the time $(t=0)$ is not the initial time of the DNS as the initial transient does not appear in these plots. The temporal oscillations of these quantities appear to last permanently.

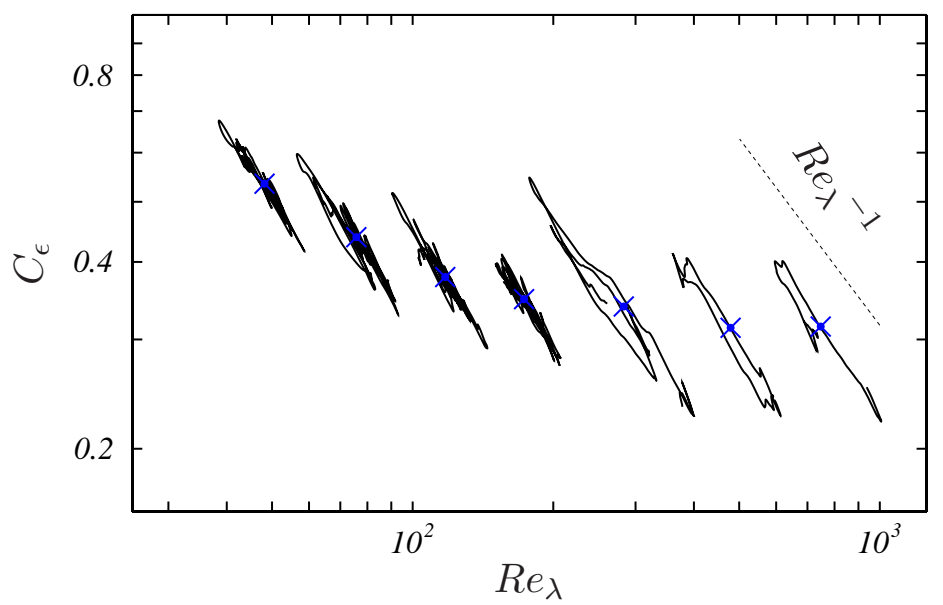

Figure 3: Instantaneous value of $C_{\epsilon}(t)$ as a function of $R e_{\lambda}(t)$ in turbulence forced by (6) for seven different values of $\nu$. Symbols $(\times)$ denote the time-averaged dissipation $\langle\epsilon\rangle$ normalized by $\left\langle u^{\prime}\right\rangle^{3} /\langle L\rangle$ as a function of $\left\langle R e_{\lambda}\right\rangle$. 


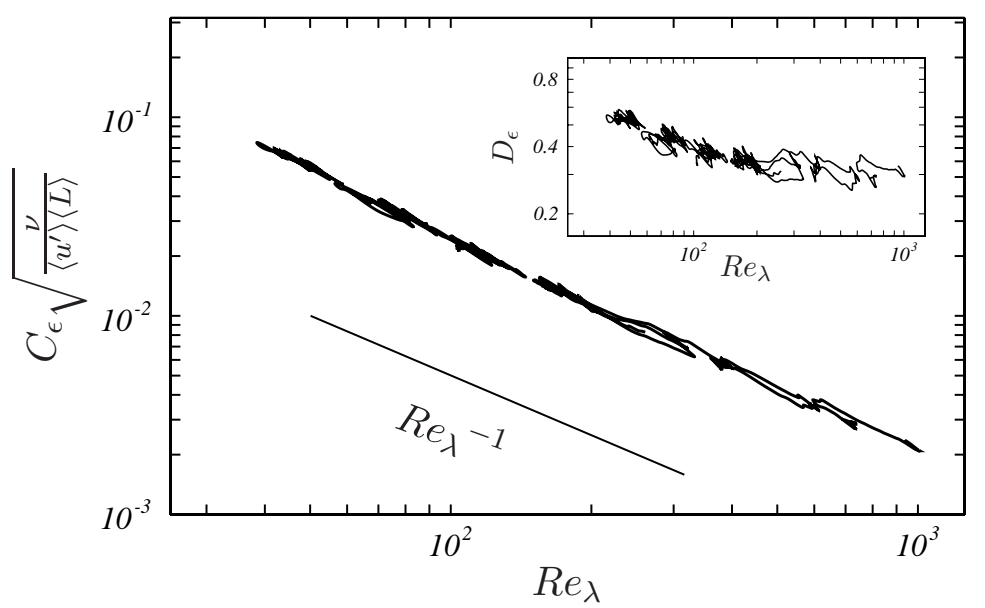

Figure 4: $C_{\epsilon}(t) / \sqrt{R e_{0}}$, where $R e_{0}=U_{0} L_{0} / \nu$ is evaluated as $R e_{0}=\left\langle u^{\prime}\right\rangle\langle L\rangle / \nu$, as a function of $R e_{\lambda}(t)$ for the forced turbulence. Insert: $D_{\epsilon}(t)$ as a function of $R e_{\lambda}(t)$.

of the temporal averages of $u^{\prime}(t)$ and $L(t)$ which makes it time-independent and representative of global forcing conditions, as it should be. We have confirmed that this quasi-periodic behavior with approximate period $20\langle T\rangle$ is always observed irrespective of $R e_{0}$. It has also been shown in Ref. [13, 14] that it is caused by energy cascade events occurring quasi-periodically. It is worth mentioning that temporal oscillations of turbulence driven by steady force is not peculiar to our system, but is observed in various flow systems (see, e.g., Ref. [15] for the interesting observation that von Kármán flow has temporal correlations of the power input lasting up to 20 eddy turnover times). It must be emphasized that the oscillations are not transient but permanent. These oscillations are manifest in Fig. 3 where, for each $R e_{0}$, the curves are not single valued but result from alternations between turbulence decay periods where $R e_{\lambda}(t)$ decays and $C_{\epsilon}(t)$ grows and turbulence build-up periods where $R e_{\lambda}(t)$ grows and $C_{\epsilon}(t)$ decays. In Fig. 3 we plot the temporal evolutions of $C_{\epsilon}(t)$ and $R e_{\lambda}(t)$ for the durations $62\langle T\rangle, 71\langle T\rangle$, $78\langle T\rangle, 74\langle T\rangle, 42\langle T\rangle, 22\langle T\rangle$ and $19\langle T\rangle$ in order of increasing $R e_{0}$. More interestingly, similarly to our turbulence decay simulations, these curves run approximately along $C_{\epsilon}(t) \propto R e_{\lambda}(t)^{-1}$ lines for every $R e_{0}$ (see Fig. 3); and furthermore, they can be made to collapse if we use the global Reynolds number $R e_{0}=U_{0} L_{0} / \nu$ where $U_{0}$ and $L_{0}$ are the temporal averages of $u^{\prime}(t)$ and $L(t)$ and plot $C_{\epsilon}(t) / \sqrt{R e_{0}}$ as a function of $R e_{\lambda}(t)$ (see Fig. 4). The insert of Fig. 4 shows $D_{\epsilon}(t)$ versus $R e_{\lambda}(t)$ and demonstrates the validity of 
(4), i.e. (1) with $p=q=1$, particularly at the higher values of $R e_{\lambda}$.

In the forced turbulence, $R e_{\lambda}$ reaches values as high as $O\left(10^{3}\right)$, and in both flows the spectrum $E(k, t)$ has a sizable intermediate wavenumber range where the wavenumber dependence is close to $k^{-5 / 3}$, particularly at the highest Reynolds numbers as shown in Fig. 5. Hence the dissipation law (4) is clearly an inertial effect and not a predominantly viscous effect. Note that here we use the word "inertial" in the sense that the inertial term in the Navier-Stokes equation dominates the viscous term, but we do not use the word in the sense that the time derivative term in (3) is negligible.

\section{Interscale energy flux law}

Our forced DNS reaches the highest $R e_{\lambda}$ values and so we use it first to evaluate $\Pi(k, t)$ at various intermediate wavenumbers $k$ for our entire $R e_{0}$ range. Examples of $\Pi(k, t)$ are shown in the inserts of Fig. 5. We define

$$
C_{\Pi}(k, t)=\frac{\Pi(k, t)}{u^{\prime}(t)^{3} / L(t)}
$$

and plot its normalized value in Fig. 6 for a representative wavenumber $k=$ $5 k_{f}$ where $k_{f}=m$ is the forcing wavenumber. Very similar results with identical conclusions are obtained for other intermediate wavenumbers such as $k=10 k_{f}$ and $k=20 k_{f}$. Except for $R e_{\lambda} \lesssim 100, C_{\Pi}(k, t)$ behaves very much like $C_{\epsilon}$ in Fig. 4. The results strongly support

$$
\Pi(k, t)=D_{\Pi}(k) U_{0} L_{0} \frac{u^{\prime}(t)^{2}}{L(t)^{2}}
$$

for a range of wavenumbers larger than $k_{f}$ and smaller than $1 / \eta$. Direct evidence of the validity of (8) at high Reynolds numbers is given in the insert of Fig. 6. The inserts of Figs. 4 and 6 show that $D_{\Pi}$ and $D_{\epsilon}$ tend to very close values at high Reynolds numbers. However this balance does not correspond to a balance between $\epsilon(t)$ and $\Pi(k, t)$ because $\partial E(k, t) / \partial t \neq 0$ and instantaneous values of $\epsilon(t)$ and $\Pi(k, t)$ are nearly never equal. In our forced turbulence, $\partial E(k, t) / \partial t$ takes both positive and negative values depending on wavenumber $k$ and time $t$, and $\epsilon(t)$ and $\Pi(k, t)$ oscillate around $\epsilon(t)=\Pi(k, t)$ (see Fig. 7). Equations (4) and (8) must therefore be understood as averages conditional on a certain value of $R e_{\lambda}$.

The situation for the decaying turbulence is simpler because $\partial E(k, t) / \partial t$ is always negative, and so is its integral over the wavenumber range $[k, \infty)$. 

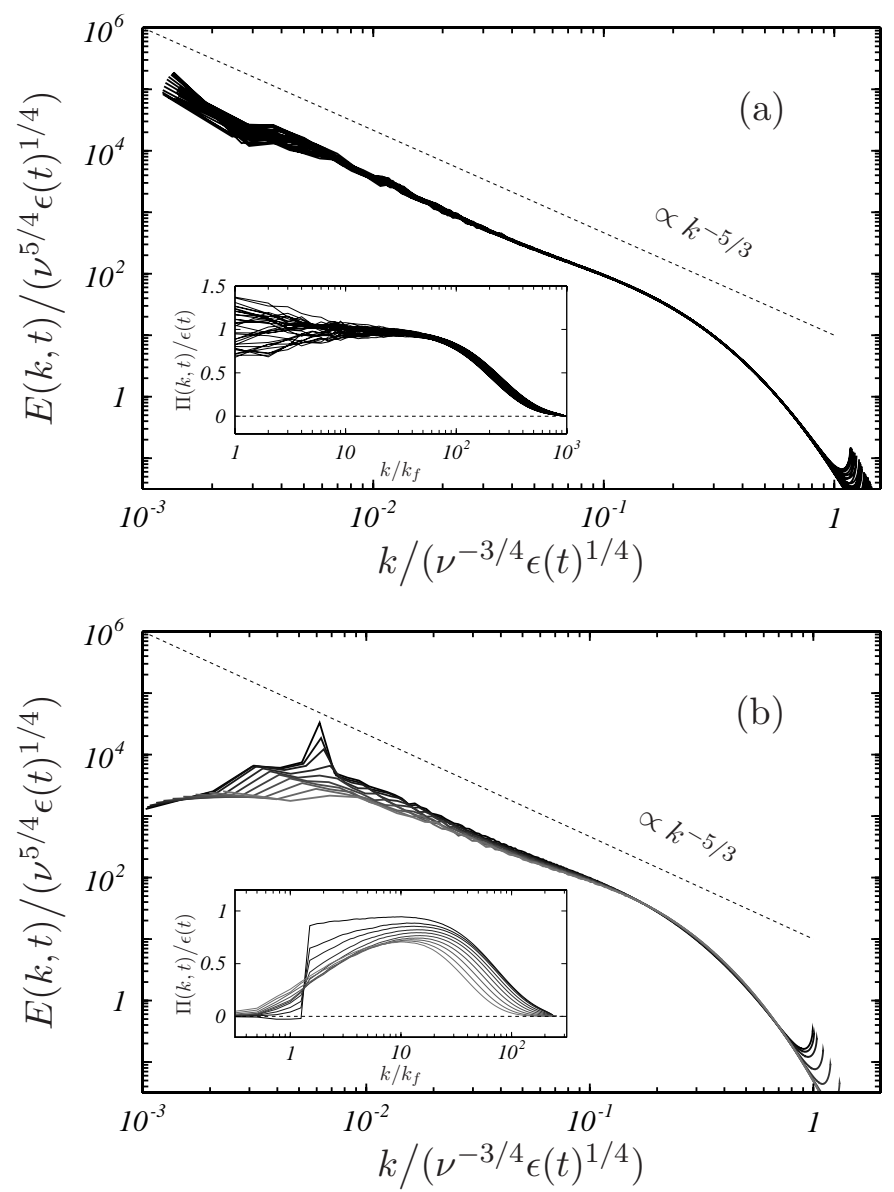

Figure 5: (a) $E(k, t)$ at 33 different times with a time interval of about $0.56\langle T\rangle$ for the highest $R e_{0}$ case of the forced turbulence. Insert: $\Pi(k, t) / \epsilon(t)$ at the same times. In agreement with Fig. 7, there are two bunches of curves in this insert corresponding to turbulence decay and buid-up periods, respectively. (b) Same as (a) but for the decaying turbulence at the highest $R e_{0} . E(k, t)$ at 10 different times with a non-dimensionalised time interval equal to 0.5 (calculated by the time-integration method given in the second paragraph of section 2). Time progresses from black to gray curves. Insert: $\Pi(k, t) / \epsilon(t)$ at the same times. The clear simultaneous validity of (4) and (8), which implies collapse of these curves in an intermediate wavenumber range, is only observed for non-dimensionalised times $\gtrsim 2$. This is because (4), i.e. (5), is slightly less well-defined for a given $R e_{0}$ in the decaying case (Fig. 1) than in our forced case, probably because of the smaller Reynolds numbers. 


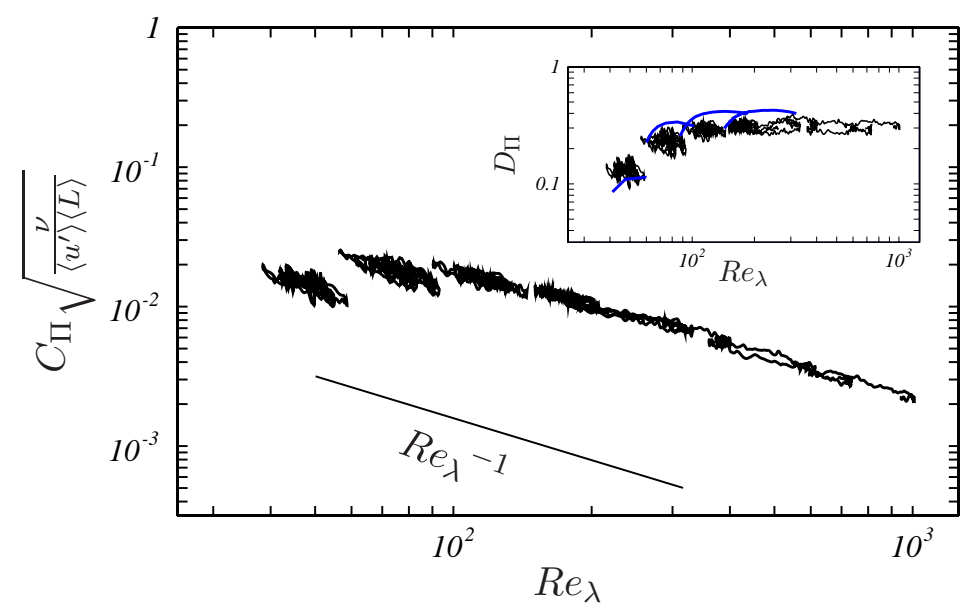

Figure 6: $C_{\Pi}(t) / \sqrt{R e_{0}}$ as a function of $R e_{\lambda}(t)$ in the forced turbulence for seven different values of $\nu$. The flux $\Pi(k, t)$ is calculated at $k=5 k_{f}$. Insert: $D_{\Pi}(t)$ as a function of $R e_{\lambda}(t)$. Black, forced turbulence; blue, decaying turbulence $\left(k=10 k_{f}\right)$.
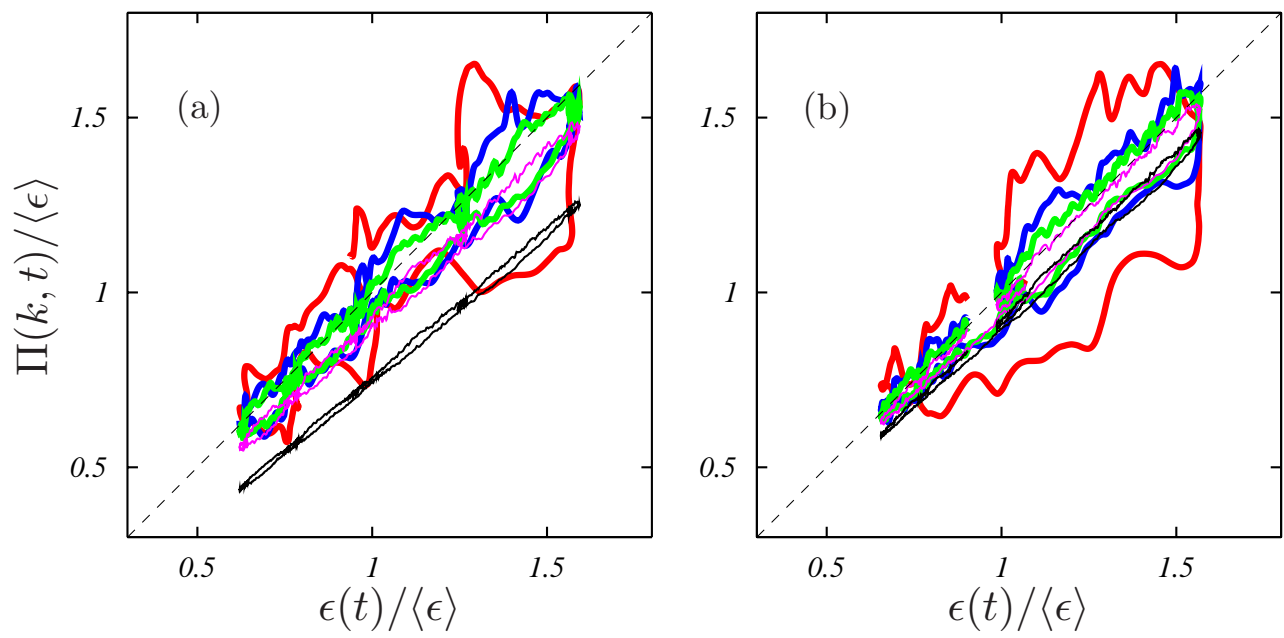

Figure 7: Energy flux $\Pi(k, t)$ as a function of $\epsilon(t)$ in the forced turbulence case for seven different values of $k$ : red line, $2 k_{f}$; blue, $5 k_{f}$; green, $10 k_{f}$; purple, $20 k_{f}$; black, $40 k_{f}$. The dashed line is $\epsilon(t)=\Pi(k, t)$. The time-averaged Taylor-length Reynolds number $\left\langle R e_{\lambda}\right\rangle$ is (a) 480 and (b) 750 . 
Therefore $\Pi(k, t)-\epsilon(t)<0$ and there is no balance between flux and dissipation. Nevertheless, $\Pi(k, t)$ obeys $(8)$ at high enough Reynolds numbers (see the blue curves in the insert of Fig. 6). Indeed our numerical results for the decaying turbulence indicate that $D_{\Pi} \neq D_{\epsilon}$ in agreement with the imbalance between $\Pi(k, t)$ and $\epsilon(t)$.

\section{Conclusion}

We have numerically investigated the energy dissipation rate $\epsilon(t)$ and the interscale energy flux $\Pi(k, t)$ in unsteady (both decaying and forced) turbulence in a periodic cube. The results for $\epsilon(t)$ are similar to observations in wind tunnel experiments [1]. The results for $\Pi(k, t)$ indicate that, at high enough Reynolds numbers, both $\Pi(k, t)$ and $\epsilon(t)$ scale as $U_{0} L_{0} u^{\prime}(t)^{2} / L(t)^{2}$. The fact that this scaling is found in both forced unsteady turbulence and freely decaying turbulence suggests general validity.

Before closing this article, it may be worth making a comment on averaging. In the freely decaying turbulence, even if we take an ensemble average over many realizations, $\partial\langle E\rangle / \partial t$ is negative and non-zero for any $k$. It is this invalidity of the Kolmogorov stationarity assumption that sets the stage for (though does not necessarily imply) the invalidity of (2). In contrast, in forced turbulence, an ensemble average, or long-time average, smooths out temporal fluctuations. Then, obviously $\mathrm{d}\langle E\rangle / \mathrm{d} t=0$, and therefore (3) leads to $\langle\Pi\rangle=\langle\epsilon\rangle$. It is also the case (ignoring intermittency corrections) that the Kolmogorov energy spectrum scaling holds for the average spectrum, i.e.

$$
\langle E\rangle \sim\langle\epsilon\rangle^{2 / 3} k^{-5 / 3}
$$

If (9) holds down to wavenumbers as small as $O(1 / L)$, then one can derive (2) for the time-averaged value $\langle\epsilon\rangle$ of the energy dissipation rate by integrating (9) over $k$. This is consistent with the observation in Fig. 3, where the symbols $(\times)$ give, for each $R e_{0}$, the values of $\langle\epsilon\rangle$ normalized by $\left\langle u^{\prime}\right\rangle^{3} /\langle L\rangle$ on the vertical axis and of $\left\langle R e_{\lambda}\right\rangle$ on the horizontal axis. The resulting curve depends only weakly on $\left\langle R e_{\lambda}\right\rangle$ and suggests a tendency towards a constant value as $\left\langle R e_{\lambda}\right\rangle \rightarrow \infty$. Both (9) and (2) are therefore applicable to time-averaged flows but not to instantaneous ones for which the Kolmogorov stationarity assumption is invalid but the different dissipation law (4) applies. It is a non-trivial observation that the scaling (4) holds for the time-varying $\epsilon(t)$ in both forced and decaying turbulence. 
The new scalings (4) and (8) are quite robust and valid at high values of $R e_{\lambda}$. These new scalings are incompatible with equilibrium turbulence. They can therefore be expected to have far-reaching consequences on turbulence theory and modeling which currently rely heavily on (2) and which are routinely applied to turbulent flows with Reynolds numbers comparable to those of our study. However, the physics behind these new scalings remain unknown at this stage, and an important task for the immediate future will be to develop a new theory of unsteady turbulence which reveals what lies behind the scalings (4) and (8).

\section{Acknowledgments}

SG and JCV respectively acknowledge the support of JSPS Grant-in-Aid for Scientific Research 25249014 and of an ERC Advanced Grant (2013-2018).

[1] J. C. Vassilicos, Dissipation in turbulent flows, Ann. Rev. Fluid Mech. 47 , in press, available online.

[2] G. I. Taylor, Statistical theory of turbulence, Proc. Roy. Soc. A 151 (1935) 421-444.

[3] A. A. Townsend, The structure of turbulent shear flow (2nd edition), Cambridge Univ. Press., 1980.

[4] B. Launder, D. B. Spalding, Mathematical Models of Turbulence, Academic Press, 1972.

[5] H. Tennekes, J. L. Lumley, A First Course in Turbulence, M.I.T. Press, Cambridge, Mass., 1972.

[6] U. Frisch, Turbulence, The Legacy of A. N. Kolmogorov, Cambridge Univ. Press, 1995.

[7] G. K. Batchelor, The theory of homogeneous turbulence, Cambridge Univ. Press., 1953.

[8] S. B. Pope, Turbulent flows, Cambridge Univ. Press., 2000. 
[9] A. N. Kolmogorov, The local structure of turbulence in incompressible viscous fluid for very large Reynolds numbers, Dokl. Akad. Nauk SSSR 30 (1941) 301-305, English translation in Proc. R. Soc. London, Ser.A 434, 9-13 (1991).

[10] W. D. McComb, A. Berera, M. Salewski, S. Yoffe, Taylor's (1935) dissipation surrogate reinterpreted, Phys. Fluids 22 (2010) 061704.

[11] P. C. Valente, R. Onishi, C. B. da Silva, Origin of the imbalance between energy cascade and dissipation in turbulence, Phys. Rev. E 90 (2014) 023003.

[12] M. Meldi, H. Lejemble, P. Sagaut, On the emergence of non-classical decay regimes in multiscale/fractal generated isotropic turbulence, J. Fluid Mech. 756 (2014) 816-843.

[13] T. Yasuda, S. Goto, G. Kawahara, Quasi-cyclic evolution of turbulence driven by a steady force in a periodic cube, Fluid Dyn. Res. 46 (2014) 061413.

[14] S. Goto, Y. Saito, G. Kawahara, Hierarchy of anti-parallel vortex tubes in turbulence at high Reynolds numbers, J. Fluid Mech. (submitted).

[15] J.-F. Pinton, P. C. W. Holdsworth, R. Labbé, Power fluctuations in a closed turbulent shear flow, Phys. Rev. E 60 (1999) R2452-R2455. 